\title{
Capsule Commentary on Shultz et al., Patient Preferences for Test Result Notification
}

\author{
Holly O. Witteman, $P h D^{1,2}$ \\ 'Department of Family and Emergency Medicine, Office of Education and Continuing Professional Development, Laval University, Québec, \\ Canada; ${ }^{2}$ Public Health and Optimal Health Practices Research Unit, Research Center of the CHU de Québec, Quebec City, Quebec, Canada.
}

$\mathrm{J}$ Gen Intern Med 30(11): 1698

DOI: $10.1007 / \mathrm{s} 11606-015-3433-0$

(c) Society of General Internal Medicine 2015

S hultz and colleagues ${ }^{1}$ report findings from a survey of patients regarding ways that they might prefer to receive a variety of laboratory test results. Responses varied between and within participants, depending on factors such as the type of test, whether the hypothetical result was normal or abnormal, and participant age.

This study is set against a background of concerns about overuse of tests, tensions between data security and user convenience, and efforts to increase patient access to health care and health data.

Overuse of tests is not routinely discussed in the context of patient access to test results, but as access expands, so will opportunities for offering information from resources such as the Choosing Wisely initiative. ${ }^{2}$

Shultz and colleagues highlight the trade-offs between user convenience and data security. Participants generally preferred "secure email" over "unsecure email," but it is unclear whether all participants understood the implications of such preferences. Survey materials described secure email as "password protected to insure confidentiality of data," somewhat glossing over encryption procedures. Addressing tensions between convenience and security is critical for realizing the potential of health information technology.

Not addressed in this paper are similar tensions between mode and timeliness of results delivery. Telephone calls and office visits were popular options. However, waiting for test results can be nerve-racking for patients, ${ }^{3}$ and preferences might shift if an appointment required waiting for weeks, whereas results were viewable on a patient portal within days.
Patient access to health data, appropriately, is increasing. Yet not everyone feels comfortable with patients directly accessing their laboratory test results. There are some legitimate concerns about the lack of interpretability of current test result formats ${ }^{4}$ or about test results causing false alarms or false comfort. However, such worries might benefit from some historical context: similar concerns were raised about home pregnancy tests nearly 35 years ago. ${ }^{5}$

As Shultz and colleagues argue convincingly, assessing patients' preferences is important for determining which technological system to prioritize for implementation. However, variability in participant responses suggests that the goal of providing test results in ways that suit patients might be best achieved by offering all feasible options and letting patients choose.

Conflict of Interest: The author has no conflict of interest with regard to any of the material in this manuscript.

Corresponding Author: Holly O. Witteman, PhD; Department of Family and Emergency Medicine, Office of Education and Continuing Professional Development Laval University, Québec, Canada (e-mail: holly.witteman@fmed.ulaval.ca).

\section{REFERENCES}

1. Shultz SK, Wu R, Matelski JJ, Lu X, Cram P. Patient Preferences for Test Result Notification. J Gen Intern Med. 2015. doi:10.1007/s11606-0153344-0.

2. McMahon LF, Beyth RJ, Burger A, Chopra V, Feldstein D, Korenstein D, et al. Enhancing Patient-Centered Care: SGIM and Choosing Wisely. J Gen Intern Med. 2013;29(3):432-3.

3. Kolata G. Sick and Scared, and Waiting, Waiting, Waiting. The New York Times. 1st ed. New York, NY; 2005 Aug 20;A1:3.

4. Zikmund-Fisher BJ, Exe NL, Witteman HO. Numeracy and literacy independently predict patients' ability to identify out-of-range test results. J Med Internet Res. 2014;16(8):e187.

5. Pregnancy testing. Br Med J. 1971;4(5785):444-5. 